\title{
Disease modifying therapies and infection risks in multiple sclerosis-a decision-making conundrum
}

\author{
Kok Pin Yong ${ }^{1,2,3}$, Ho Jin Kim $^{3}$ \\ ${ }^{1}$ Department of Neurology, National Neuroscience Institute, Singapore; ${ }^{2}$ Duke-NUS Graduate Medical School, Singapore; ${ }^{3}$ Department of \\ Neurology, Research Institute and Hospital of National Cancer Center, Goyang, Korea \\ Correspondence to: Dr. Ho Jin Kim. Department of Neurology, Research Institute and Hospital of National Cancer Center, Goyang, Korea. \\ Email: hojinkim@ncc.re.kr. \\ Provenance and Peer Review: This article was commissioned and reviewed by the Section Editor Jinming Han, MD (Department of Clinical \\ Neuroscience, Center for Molecular Medicine, Karolinska Institutet, Karolinska University Hospital, Stockholm, Sweden). \\ Comment on: Luna G, Alping P, Burman J, et al. Infection Risks Among Patients With Multiple Sclerosis Treated With Fingolimod, Natalizumab, \\ Rituximab, and Injectable Therapies. JAMA Neurol 2019. [Epub ahead of print].
}

Submitted Dec 27, 2019. Accepted for publication Jan 09, 2020.

doi: 10.21037/atm.2020.01.119

View this article at: http://dx.doi.org/10.21037/atm.2020.01.119

Multiple sclerosis (MS) is a chronic immune-mediated demyelinating and neurodegenerative disorder of the central nervous system (CNS), its natural history being commonly characterized by relapses, infrequently progressive from onset, resulting in irreversible cumulative neurological disability and impairment of quality of life. With the goals to reduce the number and severity of these clinical and radiological relapses and more recently to halt the disease activity in mind, numerous disease-modifying therapies (DMTs) have been developed over the last more than two decades. Since the approval of interferon- $\beta-1 \mathrm{~b}$ (IFN $\beta-1 \mathrm{~b}$ ) by FDA in 1993 for treatment of relapsing-remitting multiple sclerosis (RRMS), several other DMTs for MS have been introduced comprising of more injectables, oral drugs, and monoclonal antibodies (1). Although the pharmacological armamentarium for MS has expanded and is still growing, the concerns of potentially increased infection risks associated with these DMTs will inexorably be present given their immunosuppressive or immunomodulatory effects (2). These risks will need to be taken into account during riskbenefit assessment of MS therapies, in addition to other multiple factors.

As the old adage goes, prevention is better than cure. In this regard, risk mitigation strategies (RMS) play a significant role. To have RMS in place, we first need to recognize the presence of specific DMT related infections and potential risk factors for their development. These risk factors could include, but not limited to, patients' age, gender, concurrent co-morbidities, prior treatment such as chemotherapy and other immunotherapies, and body mass index, etc. In this context, the required data can be pooled from randomized controlled trials (RCTs), post-marketing surveillance (PMS), and real world evidence (RWE). RCTs are conducted in rigidly controlled settings involving highly selective population defined by strict inclusion and exclusion criteria as well as short period of follow-up. Consequentially, findings from RCTs may not be representative that of the actual entire population and potentially miss informing treatment related infection risks. As such, PMS and RWE play vital parts especially in identifying infections, which are inherently chronic and indolent and may not be observed during the typically fixed period of RCTs.

Findings from the study by Luna $\mathrm{G}$ et al., published recently in the October 2019 issue of JAMA Neurology, are thus timely and most welcome (3). Using the nationwide MS register, linked to national health care and census registries, they reviewed and compared the infection risks among Swedish patients with MS who were treated with IFN $\beta$, glatiramer (GA), fingolimod, natalizumab, or rituximab as well as among the general population. Data on 8,600 treatment episodes were retrieved from 6,421 patients and mean total treatment duration of IFN $\beta$ and GA was identified as 2.1 years, 2.7 years for fingolimod, 2.5 years for natalizumab, and 2.0 years for rituximab. The authors concluded that patients with MS who were treated with rituximab had the highest risk of serious infection, 
defined as any infection requiring hospitalization, while the lowest rates were observed in patients with IFN $\beta$ and GA. Natalizumab and fingolimod usage was not associated with significantly higher rates of infection compared to the injectables after adjusting for confounders. These findings were to some extent differed with that published by Wijnands JMA et al., whose cohort study among 6,793 patients with MS showed that higher infection risk was associated with fingolimod, dimethyl fumarate (DMF), and especially natalizumab as compared to treatment with IFN $\beta$ and GA. No patients on rituximab were included in this study (4).

Nevertheless, the findings have provided useful information regarding the relative risks of infection among the studied DMTs via utilization of RWE and it would be interesting to identify the reasons or factors for the increased infection risk associated with one drug compared to another. For example, previous reports related to rheumatoid arthritis and other non-neurological autoimmune diseases have suggested increased risk of infection and mortality rates with hypogammaglobulinemia after rituximab therapy (5). In addition, one study reported that prolonged treatment with rituximab in patients with neuromyelitis optica spectrum disorder was associated with hypogammaglobulinemia and possibly with risk of infection (6). Similar observation in MS patients treated with rituximab and ocrelizumab are also emerging (7-9). Yet, an observational report on long term safety of rituximab in rheumatoid arthritis over a period of 11 years suggested that serious infection events remained stable pre-, during, or post-development of hypogammaglobulinemia (10). As such, could hypogammaglobulinemia still be a contributing factor for the higher infection risk observed with rituximab in this study? Given the vast availability of real-world data in this study, analyzing the patients' immunoglobulin levels and delineating its correlation with rituximab as well as the associated infection risk (for instance, the lowest limit of immunoglobulin level as threshold for increased infection risk and as indicator for administration of immunoglobulin replacement therapy) may hopefully provide some answers and go a long way toward establishing RMS. This is particularly relevant and pertinent as anti-CD20 B celldepleting therapies are likely to be increasingly used as longterm treatment in patients with RRMS (ocrelizumab and rituximab) and primary progressive MS (ocrelizumab), given their demonstrated efficacy in recent pivotal trials and likely increased treatment adherence in view of their administration in single or short-intermittent courses (11-13).

Another data worth extracting from this study, if available, and potentially contributing to more effective RMS would be lymphocyte counts. In a recent review of lymphopenia and DMTs in MS, the association between lymphopenia and opportunistic infections is not clear for most of the current DMTs (14). However, we are still concerned about the potential risk of infection conferred naturally by these immunosuppressive and immunomodulatory agents administered over time, especially in light of reports on DMF-associated prolonged lymphopenia and progressive multifocal leukoencephalopathy (PML) (15). Furthermore, observation from a retrospective review showed that nearly up to one-third of 41 fingolimod-treated patients with MS in Japan was found to have marked lymphopenia within a year of treatment (16). The role of lymphopenia in this context needs to be more well-defined given its implication in decision-making, especially when it influences the decision to cease treatment. For example, discontinuing fingolimod has been linked to the accounts of rebound worsening of disease (severely increased disability and presence of multiple new lesions on MRI) $(17,18)$. Continuous monitoring of absolute lymphocyte counts from pre-commencement of therapy and beyond posttreatment hence should still be included in the RMS.

Future RMS may, not unexpectedly, incorporate genetic factors, in particular when prevalence of certain infections and their risk factors is plausibly associated with racial differences. An instance in mind concerns the reported findings of significantly higher seroprevalence of anti-JohnCunningham virus (JCV) antibodies and higher proportion of patients with elevated index among Asian (e.g., Japanese, Chinese, and Korean) patients with MS as compared to the Western cohorts (19-21). The exact reason for this apparent discrepancy is still not fully elucidated. Additionally, it appears that the prevalence of fingolimod-associated PML is significantly higher among the Japanese patients with MS (22). Further studies looking into the contribution of genetic factors in explaining these findings will be useful.

RWE such as those obtained in this study provides an opportunity to ascertain the role of increasing DMT dosages with cumulative infection risk over a long period of treatment exposure. The experience with natalizumabrelated PML serves as a good example, with longer natalizumab therapy (especially $>24$ months) identified as a risk factor involved in development of PML, in addition to presence of serum anti-JCV antibodies and previous exposure to immunosuppressive agents (23). These findings subsequently led to establishment of extensive RMS for patients on natalizumab. In the same vein, RWE should 
be continually looked into to enhance the detection of specific infections associated with specific DMT. While cryptococcal infections, varicella-zoster virus (VZV) related infection, and herpes simplex encephalitis (HSE) have been infrequently linked to fingolimod usage and only few patients on alemtuzumab have been found to develop listeriosis, tuberculosis (TB) (also observed particularly in patients treated with the anti-CD20 monoclonal antibodies), and Pnuemocystis pneumonia, it is still imperative for prescribing physicians to be aware of the possibility of these opportunistic infections arising from the specific DMTs. Targeted or specific screening for these infections can potentially result in cost-effective medical practice.

Comprehensive acquaintance with the specific treatment related infections, the extent of risk conferred by the specific drug to their development (reactivation in certain cases), and the potential risk factors for their occurrence, some of which have been discussed so far, facilitates preventative measures as part of the RMS. Appreciation of the epidemiology of infectious diseases where some infections are more prevalent in certain regions of the world would be essential e.g., screening for and treating latent $\mathrm{TB}$ infection prior to starting DMTs is especially suggested for patients residing in countries of high burden or high-risk populations. If required, infectious disease physicians are to be consulted. Practical guidelines on immunization of vaccine-preventable infections (influenza, hepatitis $B$, and VZV) have been made available recently. Vaccination should be considered during treatment discussion with patients and administered prior to initiation of DMTs, avoiding usage of live-attenuated vaccines while on DMTs and delaying them while patients are experiencing a relapse (24).

All in all, the efforts of Luna et al. in utilizing RWE in their analysis of infection risks related to different DMTs in patients with MS ought to be applauded. While questions remain regarding the roles of certain factors such as hypogammaglobulinemia, lymphopenia and perhaps even genetic predisposition in developing more specific infections with certain DMTs compared to others, future studies analyzing these and other specific factors via RWE are needed. Understanding these factors will hopefully gear us toward more effective RMS and optimize our decision making when it comes to benefit-risk ratio. Lest we forget, we shall first do no harm.

\section{Acknowledgments}

Funding: This study was supported by the Advanced
Research Center Program (NRF-2018R1A5A2023127) of the National Research Foundation of Korea.

\section{Footnote}

Conflicts of Interest: Both authors have completed the ICMJE uniform disclosure form (available at http://dx.doi. org/10.21037/atm.2020.01.119). HJK has received a grant from the National Research Foundation of Korea; received consultancy/speaker fees from Alexion, Celltrion, Eisai, HanAll BioPharma, Merck Serono, Novartis, Sanofi Genzyme, Teva-Handok, and Viela Bio; serves on a steering committee for MedImmune/Viela Bio; is a co-editor for the Multiple Sclerosis Journal and an associated editor for the Journal of Clinical Neurology. KPY has no conflicts of interest to declare.

Ethical Statement: The authors are accountable for all aspects of the work in ensuring that questions related to the accuracy or integrity of any part of the work are appropriately investigated and resolved.

Open Access Statement: This is an Open Access article distributed in accordance with the Creative Commons Attribution-NonCommercial-NoDerivs 4.0 International License (CC BY-NC-ND 4.0), which permits the noncommercial replication and distribution of the article with the strict proviso that no changes or edits are made and the original work is properly cited (including links to both the formal publication through the relevant DOI and the license). See: https://creativecommons.org/licenses/by-nc-nd/4.0/.

\section{References}

1. Tintore M, Vidal-Jordana A, Sastre-Garriga J. Treatment of multiple sclerosis - success from bench to bedside. Nat Rev Neurol 2019;15:53-8.

2. Winkelmann A, Loebermann M, Reisinger EC, et al. Disease-modifying therapies and infectious risks in multiple sclerosis. Nat Rev Neurol 2016;12:217-33.

3. Luna G, Alping P, Burman J, et al. Infection risks among patients with multiple sclerosis treated with fingolimod, natalizumab, rituximab, and injectable therapies. JAMA Neurol 2019. [Epub ahead of print].

4. Wijnands JMA, Zhu F, Kingwell E, et al. Diseasemodifying drugs for multiple sclerosis and infection risk: a cohort study. J Neurol Neurosurg Psychiatry 2018;89:1050-56. 
5. Barmettler S, Ong MS, Farmer JR, et al. Association of immunoglobulin levels, infectious risk, and mortality with rituximab and hypogammaglobulinemia. JAMA Netw Open 2018; 1:e184169.

6. Marcinnò A, Marnetto F, Valentino $\mathrm{P}$, et al. Rituximabinduced hypogammaglobulinemia in patients with neuromyelitis optica spectrum disorders. Neurol Neuroimmunol Neuroinflamm 2018;5:e498.

7. Tsao L, Otani IM, Bove R. Hypogammaglobulinemia in multiple sclerosis patients receiving disease-modifying immunomodulatory agents. J Allergy Clin Immunol 2019;143:AB16.

8. Hallberg S, Boremalm M, Evertsson B, et el. Risk of hypogammaglobulinemia in long- term treatment with rituximab in multiple sclerosis [abstract]. In: 35 th Congress of the European Committee for Treatment and Research in Multiple Sclerosis; 2019 Sept 11- 13; Stockholm, Sweden. MSJ; 2019. Abstract nr 64.

9. Derfuss T, Weber MS, Hughes R, et al. Serum immunoglobulin levels and risk of serious infection in the pivotal phase III trials of ocrelizumab in multiple sclerosis and their open- label extensions [abstract]. In: 35 th Congress of the European Committee for Treatment and Research in Multiple Sclerosis; 2019 Sept 11- 13; Stockholm, Sweden. MSJ; 2019. Abstract nr 65.

10. van Vollenhoven RF, Fleischmann RM, Furst DE, et al. Long-term safety of rituximab: Final report of the rheumatoid arthritis global clinical trial program over 11 years. J Rheumatol 2015;42:1761-6.

11. Hauser SL, Waubant E, Arnold DL, et al. B-cell depletion with rituximab in relapsing-remitting multiple sclerosis. $\mathrm{N}$ Engl J Med 2008;358:676-88.

12. Hauser SL, Bar-Or A, Comi G, et al. Ocrelizumab versus interferon beta- 1a in relapsing multiple sclerosis. N Engl J Med 2017;376:221-34.

13. Montalban X, Hauser SL, Kappos L, et al. Ocrelizumab versus placebo in primary progressive multiple sclerosis. $\mathrm{N}$ Engl J Med 2017;376:209-20.

14. Fox EJ, Buckle GJ, Singer B, et al. Lymphopenia and

Cite this article as: Yong KP, Kim HJ. Disease modifying therapies and infection risks in multiple sclerosis-a decisionmaking conundrum. Ann Transl Med 2020;8(11):722. doi: 10.21037/atm.2020.01.119
DMTs for relapsing forms of MS. Considerations for the treating neurologist. Neurol Clin Pract 2019;9:53-63.

15. Berger JR. Classifying PML risk with disease modifying therapies. Mult Scler Relat Disord 2017;12:59-63.

16. Ohtani R, Mori M, Uchida T, et al. Risk factors for fingolimod-induced lymphopenia in multiple sclerosis. Mult Scler J Exp Transl Clin 2018;4:2055217318759692.

17. Avasarala J, Jain S, Urrea-Mendoza E. Approach to fingolimod-induced lymphopenia in multiple sclerosis patients: do we have a roadmap? J Clin Pharmacol 2017;57:1415-8.

18. Faissner S, Hoepner R, Lukas C, et al. Tumefactive multiple sclerosis lesions in two patients after cessation of fingolimod treatment. Ther Adv Neurol Disord 2015;8:233-8.

19. Aoyoma S, Mori M, Uchida T, et al. Analysis of anti-JCV antibody in Japanese patients with multiple sclerosis. J Neurol Sci 2017;381:238-9.

20. Lau A, Qiu W, Kermode A, et al. High prevalence and indexes of anti-John Cunningham virus antibodies in a cohort of Chinese patients with multiple sclerosis. Mult Scler J Exp Transl Clin 2018;4:2055217318788699.

21. Kim SH, Kim Y, Jung JY, et al. High seroprevalence and index of anti-John-Cunningham virus antibodies in Korean patients with multiple sclerosis. J Clin Neurol 2019; 15:454-60.

22. Nakahara J, Tomaske L, Kume K, et al. Three cases of non-carryover fingolimod-PML: is the risk in Japan increased? Neurol Neuroimmunol Neuroinflamm 2019:6:e559.

23. Ho PR, Koendgen H, Campbell N, et al. Risk of natalizumab-associated progressive multifocal leukoencephalopathy in patients with multiple sclerosis: a retrospective analysis of data from four clinical studies. Lancet Neurol 2017;16:925-33.

24. Farez MF, Correale J, Armstrong AJ, et al. Practice guideline update summary: Vaccine preventable infections and immunization in multiple sclerosis. Neurology 2019;93:584-94. 